\title{
Identification of a missense mutation of COL3A1 in a Chinese family with atypical Ehlers-Danlos syndrome using targeted next-generation sequencing
}

\author{
WENWEN ZHANG $^{1,2 *}$, QIAN HAN ${ }^{1 *}$, MIN ZHOU $^{1}$, FENG RAN $^{1}$, \\ TONG QIAO ${ }^{1}$, LONG $\mathrm{YI}^{2}$, CHANGJIAN LIU ${ }^{1}$ and $\mathrm{ZHAO} \mathrm{LIU}^{1}$ \\ ${ }^{1}$ Department of Vascular Surgery, Nanjing Drum Tower Hospital, Nanjing University Medical School, Nanjing, \\ Jiangsu 210008; ${ }^{2}$ Center for Translational Medicine, Nanjing University Medical School, Nanjing, Jiangsu 210093, P.R. China
}

Received November 25, 2015; Accepted November 21, 2016

DOI: $10.3892 / \mathrm{mmr} .2016 .6082$

\begin{abstract}
Aortopathy represents an important cause of mortality in industrialized countries, with a number of genes identified as predispose factors. It can be difficult to identify the genetic lesions underlying this disorder, particularly when the phenotype is atypical. The present study performed targeted next-generation sequencing of 428 genes associated with cardiovascular diseases in a family with aortopathy, the proband of which presented with abdominal aortic aneurysm rupture only, with tissue fragility noted in surgery. After targeted capture, sequencing and bioinformatics analysis, a missense mutation, p.A1259T, was identified in the collagen type III $\alpha 1$ (COL3A1) gene and co-segregated with the disease in the family. Crystal structure modeling revealed abnormal hydrogen bonds generated by the mutation, which likely affected the spatial structure of the procollagen C-propeptide. Mutations in the procollagen $\mathrm{C}$-propeptide are rare and genotype-phenotype correlation may explain the atypical manifestations of affected individuals. The results of the present study suggested that targeted gene capture combined with next-generation sequencing can serve as a useful technique in the genetic diagnosis of aortopathy, particularly in the content of an atypical phenotype.
\end{abstract}

Correspondence to: Dr Zhao Liu or Dr Changjian Liu, Department of Vascular Surgery, Nanjing Drum Tower Hospital, Nanjing University Medical School, 321 Zhongshan Road, Nanjing, Jiangsu 210008, P.R. China

E-mail: liuzhao83@gmail.com

E-mail:dr_cjliu@hotmail.com

*Contributed equally

Key words: aortopathy, collagen type III $\alpha 1$ mutation, targeted next-generation sequencing, atypical phenotype

\section{Introduction}

Aortopathy, predominantly manifested as aortic aneurysm and dissection, represents an important cause of mortality in industrialized countries. An estimated $20 \%$ of patients with aortopathy have a positive family history, indicating a genetic predisposition to the disease (1). Genetics-associated aortopathy can be divided into syndromic and non-syndromic subgroups, depending on the presence or absence of manifestations in other organ systems. Well-characterized syndromic forms with aortic involvement include Marfan syndrome (OMIM 154700), vascular Ehlers-Danlos syndrome (vEDS; OMIM 130050), Loeys-Dietz syndrome (OMIM 609192) and aneurysms-osteoarthritis syndrome (OMIM 613795) (2). When an underlying gene defect is suspected to be responsible for aortopathy, identifying the causative gene is critical for patient management and genetic counseling of the family (3). Affected individuals can benefit from interval screening and timely intervention.

Familial aortopathy is an autosomal dominant condition with significant genetic heterogeneity, and mutations in $>12$ genes have been identified to account for the disorder (4). Conventional methods to identify genes underlying aortopathy depend primarily on genotype-phenotype correlation. For example, patients with ocular, skeletal and cardiovascular abnormalities are often found to harbor fibrillin 1 (1) mutations. However, the clinical manifestations vary substantially, ranging from mild to severe systemic disease (5). In addition, a significant overlap of clinical features has been observed in patients with aortopathy caused by different genes (6). As a result, Sanger sequencing of all candidate genes is required to provide a molecular diagnosis, however, the procedure is laborious and time consuming. The targeted next-generation sequencing method allows for the simultaneous assessment of multiple genes of interest and can be an efficient approach to identify the genes underlying inherited aortopathy in individuals.

Pilot investigations investigating the utilization of this method in the molecular diagnosis of patients with aortopathy have shown promising results $(7,8)$. In the present study, targeted next-generation sequencing was performed to 
elucidate the gene defects in a family with aortopathy, in which the phenotype was atypical.

\section{Materials and methods}

Ethics statement and subjects. The present study was performed in accordance with the review board of Nanjing Drum Tower Hospital (Nanjing, China), and written informed consent was obtained from all study subjects. All procedures were performed according to the tenets of the Declaration of Helsinki.

The subjects included in the present study comprised a non-consanguineous Han Chinese family with suspected inherited aortopathy from Jiangsu, China (Fig. 1A). The index case (II:1; proband) was a 48-year-old woman with a ruptured abdominal aortic aneurysm (Fig. 1B), who underwent emergency surgery for repair. During vascular prostheses replacement, blood vessel friability was noted and the procedure duration was twice that estimated. The patient developed renal failure due to this extended duration of surgery. Clinical examination revealed no pronounced craniofacial, skeletal or skin anomalies. The patient also denied any tendency to bruise. The proband's sister (II:3) reported a similar disease experience at the age of 50 years. Her mother (I:2) succumbed to mortality suddenly at the age of 52 years of an unknown cardiovascular cause. Peripheral blood $(2 \mathrm{ml})$ was collected in EDTA-coated tubes from available family members and stored at $-80^{\circ} \mathrm{C}$. Genomic DNA was extracted using standard protocols. As a control group, 100 unrelated, ethnically-matched, healthy individuals were recruited at the Center of Physical Examination of Nanjing Drum Tower Hospital.

Illumina library construction. Genomic DNA was purified and quantified using a NanoDrop 2000 spectrophotometer (Thermo Fisher Scientific, Inc., Waltham, MA, USA). The generation of a targeted Illumina library was performed according to the manufacture's protocol (MyGenostics, Inc., Beijing, China). A final library size of 350-400 bp including adaptor sequencings was selected.

Targeted capture, sequencing and bioinformatics analysis. A panel of 428 genes associated with cardiovascular diseases, including 12 known disease-causing genes [FBN1, EGF-containing fibulin-like extracellular matrix protein 2, collagen type III $\alpha 1$ (COL3A1), transforming growth factor $\beta$ (TGFB) receptor 1, TGFB receptor 2, TGFB2, TGFB3, small mothers against decapentaplegic (SMAD) 3, SMAD4, actin $\alpha 2$ smooth muscle aorta, myosin heavy chain 11 and mysosin light chain kinase], were selected using a gene capture strategy with the GenCap custom enrichment kit (MyGenostics, Inc), as previously described (9). The enriched libraries were sequenced on an Illumina Solexa HiSeq 2000 sequencer for 100 -paired reads.

Following sequencing, high-quality reads were retrieved from raw reads by filtering out low-quality reads and adaptor sequences using the Solexa QA package (10) and Cutadapt program (http://code.google.com/p/cutadapt/), respectively. Short Oligonucleotide Analysis Package (SOAP) aligner software (SOAP2.21; soap.genomics.org.cn/soapsnp.html) was then used to align the clean reads to the reference human genome (hg19) (11). Polymerase chain reaction (PCR) duplicates were removed using the Picard program (12). Subsequently, single nucleotide polymorphisms (SNPs) were determined using the SOAPsnp program (11), reads were realigned using Burrows-Wheeler Aligner (13), and the deletions and insertions (InDels) were detected using Genome Analysis Toolkit software (14). The identified SNPs and InDels were annotated using the Exome-assistant program (http://122.228.158.106/exomeassistant). MagicViewer was used to view the short read alignment, and confirm the candidate SNPs and InDels (15). Non-synonymous variants were evaluated using the four algorithms, PolyPhen (http://genetics. bwh.harvard.edu/pph2/), Sorting Intolerant From Tolerant [SIFT; (http://sift.jcvi.org/)], Protein Analysis Through Evolutionary Relationships (PANTHER; www.pantherdb.org) and Pathogenic Mutation Prediction (Pmut; http://mmb.pcb. ub.es/PMut/) to determine pathogenicity (16).

Mutation validation and analysis. Genomic DNA from all available family members were obtained for Sanger sequencing. The PCR samples were visualized on agarose gels, purified and sequenced on an ABI PRISM 3730 genetic analyzer (Applied Biosystems; Thermo Fisher Scientific, Inc.) using the terminator cycle sequencing method. Sites of variation were identified through a comparison of DNA sequences with the corresponding GenBank (www.ncbi. nlm.nih.gov) reference sequences. Possible pathogenic effects caused by the rare or novel non-synonymous SNPs were evaluated using SIFT, PolyPhen2 and MutationTaster (http://www.mutationtaster.org/). Multiple sequence alignments were performed using ClustalW2 with the default setting (http://www.ebi.ac.uk/Tools/clustalw2/).

Structure analysis. The 3D structure of the procollagen III C-propeptide (Protein Data Bank; www.rcsb.org/pdb/; reference, 4AE2) was used to perform in silico analysis. The crystal structures of the mutant proteins were predicted using the SWISS-MODEL online server (17) and displayed using PyMol software (version 1.5; www.pymol.org).

\section{Results}

Targeted next-generation sequencing. The present study performed targeted capture and next-generation sequencing of 428 genes implicated in cardiovascular diseases. The average sequencing depth for the targeted regions was $\sim 328 \mathrm{X}$. The sample had $93.40 \%$ coverage of the targeted regions. There was 91.20 and $87.50 \%$ coverage of the targeted exons for $10 \mathrm{X}$ and 20X, respectively. A total of 782 variants were identified in the sample. Among these, 394 missense, nonsense, splicing variants and coding InDels were identified, which more likely to be pathogenic. This was narrowed down to 41 by excluding variants reported in the dbSNP138 (www.ncbi.nlm.nih. gov/SNP/), 1000 Genomes Project (www.1000genomes.org), Exome Sequencing Project (evs.gs.washington.edu/EVS/) and local dataset with allele frequencies $>0.01$. This was further narrowed down to 15 by filtering benign variants predicted the by four algorithms, PolyPhen, SIFT, PANTHER and Pmut. A missense variant, c.3775G $>$ A (p.A1259T) in exon 48 of COL3A1 (NM_000090), was most likely disease-causing in this family, as the mutated gene is known to be involved in 
Table I. Number of variants present in the proband (II:1) at different stages of the filtering process.

\begin{tabular}{lr} 
Filter stage & $\mathrm{n}$ \\
\hline Total variants identified & 782 \\
Non-synonymous variants & 394 \\
Variants with allele frequencies $\leq 0.01$ & 41 \\
Pathogenic variants predicted by algorithms & 15 \\
Variants in disease-causing genes of aortopathy & 1 \\
\hline
\end{tabular}
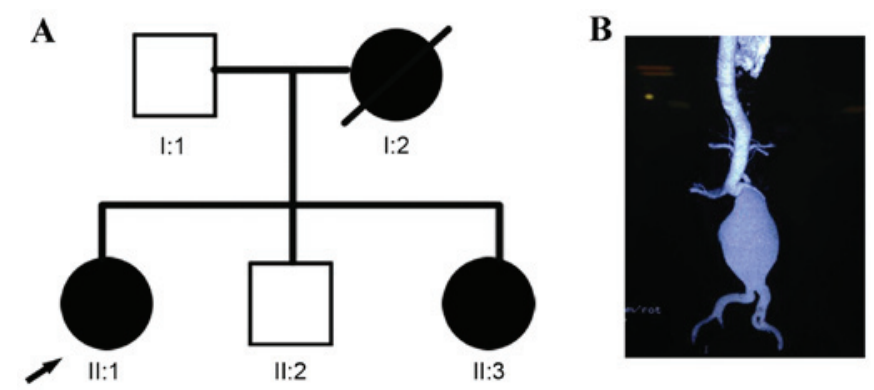

Figure 1. Pedigree and aortopathy. (A) Pedigree of the proband. Square symbols, males; round symbols, females; filled symbols, affected; unfilled symbols, unaffected; diagonal lines, deceased; arrow, proband. (B) Three-dimensional computed tomography-angiography showed a ruptured abdominal aorta aneurysm (magnification, x10).
$\mathbf{A}$

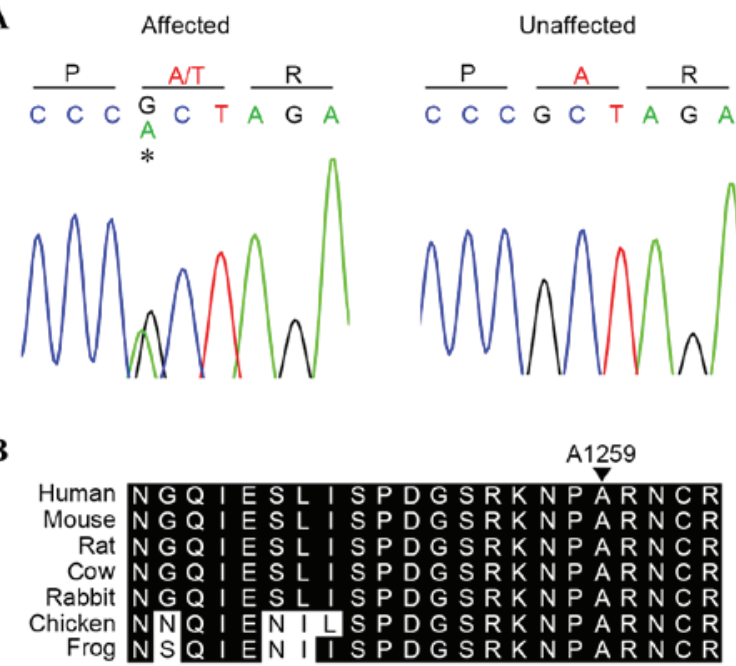

Figure 2. Heterozygous missense mutation at a conserved position in COL3A1. (A) Sanger sequence of codons 1,258-1,260 of COL3A1 in affected and unaffected individuals. Encoded amino acids are shown in single-letter code (*mutated residue). (B) Conservation of A1259 among species. The amino acid sequence of segment 1,242-1,263 of human COL3A1 is shown and compared with the corresponding sequence in other species. Positions identical to human are highlighted in black. A1259 was completely conserved across all species examined. COL3A1, collagen type III $\alpha 1$; P, proline; A alanine; $\mathrm{T}$, threonine; $\mathrm{R}$, arginine.

inherited aortopathy (Table I). For this site, 51\% (193/377) reads supported for G, whereas 49\% (184/377) reads supported for A.

Mutation validation. To confirm the above findings, the present study genotyped four available members of the family

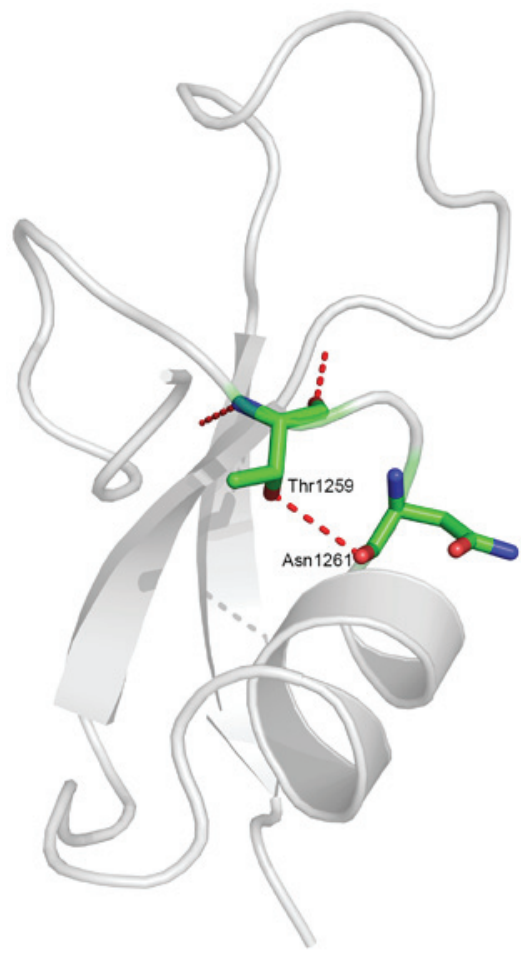

Figure 3. Predicted crystal structure. Structure modeling revealed the generation of a novel hydrogen bond between the mutated threonine at residue 1,259 and asparagine at residue 1,261.

(two affected, two unaffected) to identify the c.3775G $>$ A (p.A1259T) variant by Sanger sequencing (Fig. 2A). The affected individuals (II:1 and II:3) were found to harbor the mutation, suggesting cosegregation of the mutation with development of the disease. The sequencing results of those unaffected (I:1 and II:2) excluded the presence of the mutation, suggesting that the mutation may have been inherited from the proband's affected mother.

The variant occurred in a conserved amino acid (Fig. 2B), and was predicted to have a functional effect by SIFT, PolyPhen2 and MutationTaster. In addition, the mutation was not found on the 200 unrelated chromosomes from ethnically matched controls.

Structure analysis. The altered residue was located in the procollagen C-propeptide region, the stability of which is crucial in procollagen assembly. The missense mutation resulted in a substitution of hydrophobic alanine by hydrophilic threonine at a conserved site. Structure modeling demonstrated the generation of a novel hydrogen bond between the mutated threonine at residue 1,259 and asparagine at residue 1,261 (Fig. 3). This was likely to alter the spatial conformation of the region, thus leading to production of abnormal protein.

\section{Discussion}

vEDS is a rare autosomal dominant disorder of connective tissue owing to mutations of the COL3A1 gene encoding type III procollagen, resulting in deficiency of collagen III protein (18). Type III collagen is a major component of the skin, blood vessels and hollow organ walls. This explains why patients affected by vEDS are at increased risk of vascular, 
intestinal or uterine rupture $(19,20)$. Due to these life-threatening complications, the survival rates of affected patients are significantly reduced. The first major complication, namely vascular or internal organ rupture, occurs in $25 \%$ of patients by the age of 20 years and in $>80 \%$ of patients by the age of 40 years, with a median survival duration of 48 years (19). Therefore, early diagnosis is necessary for timely precaution measures to avoid such outcomes. Currently, vEDS is diagnosed on the basis of the presence of at least two of the following major diagnostic criteria: Characteristic facial appearance, tendency to bruise easily, translucent skin with visible veins, history of arterial, intestinal or uterine rupture. Collagen protein analysis of cultured fibroblasts or identification of mutations in the COL3A1 gene is required to achieve an ultimate diagnosis. The proband in the present study presented with minimal signs of vEDS, precluding from performing direct sequencing of the COL3A1 gene. In accordance with previously published data, the present study further supported that vEDS is a heterozygous disease with marked variability of clinical manifestations $(21,22)$.

To date, $>700$ COL3A1 mutations have been identified in vEDS cohorts (23). Almost two thirds of the mutations are heterozygous missense substitutions affecting one of the glycine residues of the (Gly-X-Y) repeat within the triple helical domain. One third of the mutations are spice-site variants causing exon skipping. These two types of mutations act in a dominant-negative manner, resulting in abnormal procollagen peptide production. The remaining COL3A1 mutations are nonsense or frameshift mutations, which cause premature termination of translation, leading to haplo-insufficiency of normal protein. Haplo-insufficiency mutations have been reported to delay the onset of complications and increase life expectancy, compared with dominant-negative mutations $(24,25)$. Despite the wide distribution regarding COL3A1 mutations, variants affecting the C-propeptide region are rare. Frank et al (26) previously categorized COL3A1 variants into five groups: i) Glycine substitutions, ii) splice-site and in-frame insertions-deletions, iii) variants leading to haplo-insufficiency, iv) non-glycine missense variants within the triple helix and v) non-glycine missense variants or in-frame InDels in the $\mathrm{N}$ - or C-terminal of the protein (26). It was found that patients with variants leading to haplo-insufficiency, non-glycine missense variants within the triple helix or non-glycine missense variants or in-frame InDels in the $\mathrm{N}$ - or C-terminal of the protein had a less typical phenotype and prominent absence of digestive complication. In line with these observations, the complication of the proband in the present study occurred at relatively late age and was limited entirely to vascular events. These findings provided further evidence that COL3A1 mutations located in the $\mathrm{C}$-termini had a milder course of the disease and fewer characteristic features.

Due to the absence of prevalent diagnostic criteria for patients with vEDS, individuals harboring this specific type of mutation are unlikely to undergo genetic analysis in clinical settings. This explains the scarcity of mutations reported in the C-propeptide region. Previously, targeted region capture combined with next-generation sequencing was successfully utilized to uncover pathologic variants of COL3A1 in individuals with aortopathy with unsuspected vEDS (7,27). As with the proband in the present study, they were subject to targeted sequencing primarily based on aortic phenotypes, rather than other syndromic manifestations. They did not present with the skin or facial features characteristic of vEDS. Therefore, targeted next-generation sequencing has been suggested as a useful diagnostic tool in patients with arotopathy, particularly in those with an absence of characteristic phenotypes. Of note, one patient was found to have unusual tissue fragility in the previous study, which aligned with the findings of the present study and several previous studies $(22,28)$. Tissue fragility may be a consequence of collagen abnormality, indicating the presence of mutations in genes encoding collagen $(22,29)$.

In conclusion, the present study identified a missense mutation in COL3A1 in a family with aortopathy by performing targeted next-generation sequencing. Despite the lack of characteristic vEDS manifestations, co-segregation analysis, the absence in public variation databases, conservation scores, bioinformatics prediction and alteration in crystal structure all provided support for the pathogenic role of the variant. The results of the present study suggested that targeted gene capture combined with next-generation sequencing serves as a useful technique in the genetic diagnosis of aortopathy, particularly in the content of atypical phenotype.

\section{Acknowledgements}

This study was supported by the Nanjing Municipal Science and Technology Commission (grant no. BL2012035), the Medical Science and Technology Development Foundation, Nanjing Department of Health (grant no. YKK13085), and the Natural Science Foundation of Jiangsu Province, China (grant no. BK20140103).

\section{References}

1. Elefteriades JA and Pomianowski P: Practical genetics of thoracic aortic aneurysm. Prog Cardiovasc Dis 56: 57-67, 2013.

2. Jondeau $\mathrm{G}$ and Boileau C: Familial thoracic aortic aneurysms. Curr Opin Cardiol 29: 492-498, 2014.

3. Erbel R, Aboyans V, Boileau C, Bossone E, Bartolomeo RD, Eggebrecht H, Evangelista A, Falk V, Frank H, Gaemperli O, et al: 2014 ESC guidelines on the diagnosis and treatment of aortic diseases: Document covering acute and chronic aortic diseases of the thoracic and abdominal aorta of the adult. The task force for the diagnosis and treatment of aortic diseases of the European society of cardiology (ESC). Eur Heart J 35: 2873-2926, 2014.

4. Pyeritz RE: Heritable thoracic aortic disorders. Curr Opin Cardiol 29: 97-102, 2014.

5. MilewiczDM, Chen H,ParkES, Petty EM,ZaghiH, Shashidhar G, Willing $M$ and Patel V: Reduced penetrance and variable expressivity of familial thoracic aortic aneurysms/dissections. Am J Cardiol 82: 474-479, 1998.

6. Isselbacher EM, Lino Cardenas CL and Lindsay ME: Hereditary influence in thoracic aortic aneurysm and dissection. Circulation 133: 2516-2528, 2016.

7. Proost D, Vandeweyer G, Meester JA, Salemink S, Kempers M, Ingram C, Peeters N, Saenen J, Vrints C, Lacro RV, et al: Performant mutation identification using targeted next generation sequencing of 14 thoracic aortic aneurysm genes. Hum Mutat 36: 808-814, 2015.

8. Ziganshin BA, Bailey AE, Coons C, Dykas D, Charilaou P, Tanriverdi LH, Liu L, Tranquilli M, Bale AE and Elefteriades JA: Routine genetic testing for thoracic aortic aneurysm and dissection in a clinical setting. Ann Thorac Surg 100: 1604-1611, 2015.

9. Wu J, Matthaei H, Maitra A, Dal Molin M, Wood LD, Eshleman JR, Goggins M, Canto MI, Schulick RD, Edil BH, et al: Recurrent GNAS mutations define an unexpected pathway for pancreatic cyst development. Sci Transl Med 3: 92ra66, 2011. 
10. Cox MP, Peterson DA and Biggs PJ: SolexaQA: At-a-glance quality assessment of illumina second-generation sequencing data. BMC Bioinformatics 11: 485, 2010.

11. Li R, Yu C, Li Y, Lam TW, Yiu SM, Kristiansen K and Wang J: Soap2: An improved ultrafast tool for short read alignment Bioinformatics 25: 1966-1967, 2009.

12. Li H, Handsaker B, Wysoker A, Fennell T, Ruan J, Homer N, Marth G, Abecasis G and Durbin R; 1000 Genome Project Data Processing Subgroup: The sequence alignment/map format and SAMtools. Bioinformatics 25: 2078-2079, 2009.

13. Li H and Durbin R: Fast and accurate short read alignment with burrows-wheeler transform. Bioinformatics 25: 1754-1760, 2009

14. McKenna A, Hanna M, Banks E, Sivachenko A, Cibulskis K, Kernytsky A, Garimella K, Altshuler D, Gabriel S, Daly M and DePristo MA: The genome analysis toolkit: A MapReduce framework for analyzing next-generation DNA sequencing data. Genome Res 20: 1297-1303, 2010.

15. Hou H, Zhao F, Zhou L, Zhu E, Teng H, Li X, Bao Q, Wu J and Sun Z: Magicviewer: Integrated solution for next-generation sequencing data visualization and genetic variation detection and annotation. Nucleic Acids Res 38 (Web Server issue): W732-W736, 2010.

16. Jin ZB, Mandai M, Yokota T, Higuchi K, Ohmori K, Ohtsuki F, Takakura S, Itabashi T, Wada Y, Akimoto M, et al: Identifying pathogenic genetic background of simplex or multiplex retinitis pigmentosa patients: A large scale mutation screening study. J Med Genet 45: 465-472, 2008.

17. Arnold K, Bordoli L,Kopp J and Schwede T:The SWISS-MODEL workspace: A web-based environment for protein structure homology modelling. Bioinformatics 22: 195-201, 2006.

18. Beighton P, De Paepe A, Steinmann B, Tsipouras $P$ and Wenstrup RJ: Ehlers-danlos syndromes: Revised nosology, villefranche, 1997. Ehlers-Danlos National Foundation (USA) and Ehlers-Danlos Support Group (UK). Am J Med Genet 77: 31-37, 1998

19. Pepin M, Schwarze U, Superti-Furga A and Byers PH: Clinical and genetic features of Ehlers-Danlos syndrome type iv, the vascular type. N Engl J Med 342: 673-680, 2000.

20. Oderich GS, Panneton JM, Bower TC, Lindor NM, Cherry KJ, Noel AA, Kalra M, Sullivan T and Gloviczki P: The spectrum, management and clinical outcome of Ehlers-Danlos syndrome type iv: A 30-year experience. J Vasc Surg 42: 98-106, 2005.
21. Jorgensen A, Fagerheim T, Rand-Hendriksen S, Lunde PI, Vorren TO, Pepin MG, Leistritz DF and Byers PH: Vascular Ehlers-Danlos Syndrome in siblings with biallelic COL3A1 sequence variants and marked clinical variability in the extended family. Eur J Hum Genet 23: 796-802, 2015.

22. Wendorff H, Pelisek J, Zimmermann A, Mayer K, Seidel H, Weirich G, Hausser I, Siegel C, Zernecke A and Eckstein HH: Early venous manifestation of Ehlers-Danlos syndrome type iv through a novel mutation in COL3A1. Cardiovasc Pathol 22: 488-492, 2013.

23. Shalhub S, Black JH III, Cecchi AC, Xu Z, Griswold BF, Safi HJ, Milewicz DM and McDonnell NB: Molecular diagnosis in vascular Ehlers-Danlos syndrome predicts pattern of arterial involvement and outcomes. J Vasc Surg 60: 160-169, 2014.

24. Leistritz DF, Pepin MG, Schwarze U and Byers PH: COL3A1 haploinsufficiency results in a variety of Ehlers-Danlos syndrome type IV with delayed onset of complications and longer life expectancy. Genet Med 13: 717-722, 2011.

25. Pepin MG, Schwarze U, Rice KM, Liu M, Leistritz D and Byers PH: Survival is affected by mutation type and molecular mechanism in vascular Ehlers-Danlos syndrome (EDS type IV). Genet Med 16: 881-888, 2014.

26. Frank M, Albuisson J, Ranque B, Golmard L, Mazzella JM, Bal-Theoleyre L, Fauret AL, Mirault T, Denarié N, Mousseaux E, et al: The type of variants at the COL3A1 gene associates with the phenotype and severity of vascular Ehlers-Danlos syndrome. Eur J Hum Genet 23: 16575-1664, 2015.

27. Campens L, Callewaert B, Muino Mosquera L, Renard M, Symoens S, De Paepe A, Coucke P and De Backer J: Gene panel sequencing in heritable thoracic aortic disorders and related entities: Results of comprehensive testing in a cohort of 264 patients. Orphan J Rare Dis 10: 9, 2015.

28. Abayazeed A, Hayman E, Moghadamfalahi M and Cain D: Vascular type Ehlers-Danlos syndrome with fatal spontaneous rupture of a right common iliac artery dissection: Case report and review of literature. J Radiol Case Rep 8: 63-69, 2014.

29. Monroe GR, Harakalova M, van der Crabben SN, Majoor-Krakauer D, Bertoli-Avella AM, Moll FL, Oranen BI, Dooijes D, Vink A, Knoers NV, et al: Familial Ehlers-Danlos syndrome with lethal arterial events caused by a mutation in COL5A1. Am J Med Genet A 167: 1196-1203, 2015. 